\title{
Dissemination of Cardiopulmonary Resuscitation Training for Nurses Treating Coronavirus Disease-2019 Patients: A Single-arm Pre-experimental Study
}

\author{
${\text { Poonam Joshi }{ }^{1} \odot \text {, Smita Das }}^{2} \oplus$, Merin Thomas ${ }^{3} \odot$, Shashi Mawar ${ }^{4} \odot$, Rakesh Garg ${ }^{5} \odot$, Ahamadulla Shariff ${ }^{6} \odot$, Lakshmanan \\ Gopichandran๑
}

\begin{abstract}
Introduction: Providing cardiopulmonary resuscitation (CPR) to a coronavirus disease-2019 (COVID-19) patient is challenging for the healthcare providers (HCP). COVID-19 cases have specific CPR requirements, which differ from standard resuscitation efforts.

Objective: This paper aims to evaluate whether online learning can be an effective methodology for imparting information on the management of cardiopulmonary arrest in COVID-19 patients due to a novel virus unknown to HCP based on evaluation of knowledge acquisition and satisfaction of the nurses in the new area of medicine and virology.

Methodology: In a single-arm, one group before and after design, from a cohort of 160 nurses trained in comprehensive cardiac life support (CCLS) formulated by the Indian Resuscitation Council (IRC), 73 nurses participated in the study. After obtaining informed consent through the email from the nurses, baseline data including demographic profile and knowledge related to CPR in COVID-19 patients were collected. An online intervention spread over 1 week was given using a validated e-learning module. The online intervention was found to be effective (preand post-intervention knowledge score $13.65 \pm 3.01$ vs $19.92 \pm 1.94, p=0.001$ ). The majority of nurses were highly satisfied with the content and the training methodology $(37.23 \pm 4.70)$.

Conclusion: A well-structured, online study material can be used in imparting knowledge and demonstrating the basic and essential skills to nurses, required for giving CPR to COVID-19 patients.
\end{abstract}

Keywords: Cardiac arrest, Cardiopulmonary resuscitation, Comprehensive cardiac life support, COVID-19, Online method of learning. Indian Journal of Critical Care Medicine (2022): 10.5005/jp-journals-10071-24128

\section{BACKGROUND}

Providing cardiopulmonary resuscitation (CPR) to a cardiac arrested patient with coronavirus disease-2019 (COVID-19) is a challenging task for the healthcare providers (HCP) due to an inherent occupational hazard of getting contaminated. ${ }^{1} \mathrm{HCP}$ is at risk of acquiring COVID-19 either due to the inhalation of aerosols or contact with fomites. ${ }^{2,3}$ The COVID-19 has specific CPR requirement, which differ from standard resuscitation efforts. ${ }^{1}$ Nurses, as the key member of a resuscitation team, must understand the new resuscitation guidelines for cardiac arrested COVID-19 to explicitly use them in patient care. The recent development in technologies has provided globally new platforms for promoting advanced learning in medical and nursing education addressing to needs of the HCP, which is as effective as traditional learning. . $^{3,4}$

The techniques in CPR are lifesaving. Disseminating information about the novel virus to HCP would undoubtedly leave an impact on novel knowledge and understanding in managing COVID patients for better predicted outcomes. Building the acumen and specialization in nursing skills would provide a tremendous benefit to nurses but also to the profession at large, their institution, and the overall building of the knowledge base that is currently under construction in the detection and management of COVID-19 patients. The objectives of the study were to disseminate the information on management of cardiac arrest in COVID-19 patients, nurses' engagement in learning, professional interaction, and usefulness in teaching for mentors.
${ }^{1,2,4,7}$ College of Nursing, AlIMS, New Delhi, India

${ }^{3}$ Department of Nursing Services, AlIMS, New Delhi, India

${ }^{5}$ Department of Onco-Anaesthesia and Palliative Medicine, AlIMS, New Delhi, India

${ }^{6}$ Computer Facility, AllMS, New Delhi, India

Corresponding Author: Rakesh Garg, Department of OncoAnaesthesia and Palliative Medicine, AlIMS, New Delhi, India, Phone: +91 9810394950, e-mail: drrgarg@hotmail.com

How to cite this article: Joshi P, Das S, Thomas M, Mawar S, Garg R, Shariff $A$, et al. Dissemination of Cardiopulmonary Resuscitation Training for Nurses Treating Coronavirus Disease-2019 Patients: A Single-arm Pre-experimental Study. Indian J Crit Care Med 2022;26(3): 327-330.

Source of support: Nil

Conflict of interest: None

\section{Materials and Methods}

In a preexperimental study, a cohort of 160 nurses trained in CCLS, working in various departments of the hospital, were contacted and explained about the study. These nurses were already trained in providing basic and comprehensive life support to the adult patients at the time of cardiac arrest 1 year back. The study was approved by the institute ethics committees (IEC/NP-541/2020) and registered on CTRI (REF/2020/05/028045, September 24, 2020). The study was conducted in October, 2021, when the COVID

(O) The Author(s). 2022 Open Access This article is distributed under the terms of the Creative Commons Attribution 4.0 International License (https://creativecommons. org/licenses/by-nc/4.0/), which permits unrestricted use, distribution, and non-commercial reproduction in any medium, provided you give appropriate credit to the original author(s) and the source, provide a link to the Creative Commons license, and indicate if changes were made. The Creative Commons Public Domain Dedication waiver (http://creativecommons.org/publicdomain/zero/1.0/) applies to the data made available in this article, unless otherwise stated. 
cases were on peak. We hypothesized that the CPR training for nurses treating COVID-19 patients would augment the knowledge levels in the body of nursing with the institution at large. After acquiring the knowledge, the nurses would be further able to protect themselves from contracting the infection while looking COVID-19 patients.

\section{Intervention}

The 7-day online self-paced asynchronous training was imparted using a training module prepared by the CCLS master trainers consisting of a brief handout on revised standard CPR guidelines in context to COVID-19 ${ }^{1}$ and 12 uploaded webinars with embedded videos, and resource material in pdf form, highlighting the knowledge and demonstration of the basic and essential skills required for managing a cardiac arrested COVID-19 patient. The basic skills included: (i) hand hygiene; (ii) donning and doffing; (iii) disinfection and sterilization; and (iv) clinical waste management. Topics covered as part of the essential skills included the management of cardiac arrested COVID-19 patient showing the first step of checking the response to the last step of postresuscitation care. The content of CPR training was independently validated by a diverse group of experts namely cardiologists, anesthesiologist, and ICU nurses and nurse educators having more than 10 years of experience in the field. The participants had access to the modules (10 minutes duration each) at their convenience during the training, who were advised to watch the webinars repeatedly sequentially for better understanding. Two online interactive sessions via the Google meets were conducted for the participants to orient about the training program, the process, expected outcomes, and post-program evaluation in the beginning. A WhatsApp group was created as help section to communicate with the nurses. The developers had integrated the intervention within the program based on the anticipated needs of the learners. The participants were also requested to post their queries and clarification in the WhatsApp group. The researcher had access to the total login times of the participants. Another online meeting was conducted at the end of the training program to clear the doubts of the participants.

\section{Tools for Data Collection}

Baseline data were collected using a prevalidated structured demographic sheet and knowledge questionnaire having 25 MCQ items (developed from a published resource material using an e-learning platform) linked Google form. ' A score of "1" was given for correct response and " 0 " for an incorrect response, with a maximum possible score of 25 . The questionnaire was based on the objectives and learning goals. At the end of the intervention, the knowledge of the nurses was assessed again. As part of the secondary outcome, the satisfaction of nurses toward online learning of new CPR guidelines in terms of content coverage and used training methodology was assessed on five-point Likert scale [strongly agree (5), agree (4), uncertain (3), disagree (2), strongly disagree (1)] using nine items with the maximum possible score of 45. The satisfaction scores were categorized as very high $>40$, high $35-40$, medium 30 to $<35$, low $<30$.

\section{Statistical Analysis}

In the absence of a similar study, a pilot study was conducted to calculate the sample size. The mean knowledge score of 10 nurses was $11.93 \pm 3.26$. It was assumed that raising the mean knowledge score by $4-5$ units would be the reflection of improved knowledge. The sample size was calculated to be 14 . Due to the availability of conventionally CCLS-trained nurses, all available 73 nurses were enrolled in the study. The data were obtained in an Excel sheet and analyzed using SPSS 22.0 using descriptive statistics. For drawing the inference, a paired " $t$ " test was used to compare the knowledge scores before and after the intervention. A $p$-value of less than 0.05 was taken as significant.

\section{Results}

\section{Demographic and Baseline Data}

A total of 73 nurses consented to participate in the study (Flowchart 1, Consort Diagram). The majority of nurses were female $(n=65)$ with a mean age of $29.9 \pm 6.14$ years, residing in the urban area. Majority of nurses had Bachelor of Science (BSc) Nursing as their professional qualification (56) with the median professional experience of 4 (2-9) years. The majority of nurses were working in the medical surgical wards (47) followed by medical-surgical ICU (16) and high dependency unit (HDU) (6), and OT (4). Majority of nurses (70) neither had any experience of working in COVID-19 wards/ICU nor any previous experience of participating in an online training program (Table 1).

The mean knowledge scores of the nurses were $13.65 \pm 3.01$. There was not much diverse level of CPR understanding among the group (Table 2).

The training program was organized through an earlier established e-learning platform provided by the institute, without any preexisting template. All the participants at the time of

\section{Flowchart 1: Consort diagram}

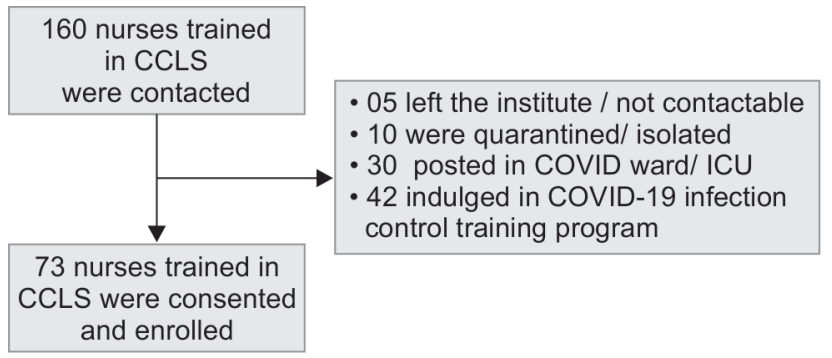

Table 1: Demographic profile of nurses $(n=73)$

\begin{tabular}{lc}
\hline Variable & $F(\%)$ \\
\hline Age $^{*}$ & $29.9 \pm 6.14$ \\
Gender (Female:Male) & $65: 8$ \\
Residence (Rural:Urban) & $9: 64$ \\
Professional qualification & $8: 56: 9$ \\
(GNM:BSc Nursing:MSc Nursing) & \\
Computer literacy & 73 \\
Present area of work (ICU:Ward:HDU:OT) & $16: 47: 6: 4$ \\
Total experience & $4(2-9)^{* *}$ \\
Experience in the present area** & $1(1-2.8)$ \\
Experience of working in COVID ward/ICU & $3: 70$ \\
Previous experience of online learning & 0 \\
Assisted in CPR of COVID-19 patient & $0(0-1)$ \\
Successful resuscitation in COVID-19 patient & $0(0-0)$ \\
Source of information about CPR in COVID-19 & $9: 13: 14: 37$ \\
patient (Journal:Online class:WhatsApp:Official & \\
notification) & \\
\hline
\end{tabular}

$\overline{\mathrm{ICU}}$, intensive care unit; $\mathrm{HDU}$, high dependency unit; OT, operation theater 
Table 2: Knowledge and satisfaction of nurses with online method of learning

\begin{tabular}{|c|c|c|c|c|c|}
\hline & Pre-intervention & Post-intervention & $\begin{array}{c}\text { Mean } \\
\text { difference }\end{array}$ & $\mathrm{Cl}$ & $p$ value \\
\hline Knowledge & $13.65 \pm 3.01$ & $19.92 \pm 1.94$ & 6.27 & $7.19-5.34$ & 0.001 \\
\hline Satisfaction & $f(\%)$ & & & & \\
\hline \multicolumn{6}{|l|}{ Categories } \\
\hline \multirow{3}{*}{$\begin{array}{l}\text { Excellent }(>40) \\
\text { Good }(35-40) \\
\text { Fair }(<35)\end{array}$} & $34(46.57)$ & $37.23 \pm 4.7$ & - & & \\
\hline & $15(20.55)$ & & & & \\
\hline & $24(32.88)$ & & & & \\
\hline
\end{tabular}

Paired " $t$ " test, $p^{*}<0.05$

Table 3: Satisfaction of nurses with online learning of updated CPR guidelines $(n=73)$

\begin{tabular}{|c|c|c|}
\hline SNo. & Statement & Median (IQR) \\
\hline \multicolumn{3}{|c|}{ About the training module } \\
\hline 1. & $\begin{array}{l}\text { Webinars were comprehensive in providing } \\
\text { information related to new guidelines of } \\
\text { CCLS }\end{array}$ & $4.5(4-5)$ \\
\hline 2. & $\begin{array}{l}\text { All videos and webinars could be } \\
\text { understood well }\end{array}$ & $5(4-5)$ \\
\hline 3. & $\begin{array}{l}\text { It was easy to understand the newer } \\
\text { guidelines of CPR on e-platform }\end{array}$ & $4(3-5)$ \\
\hline 4. & $\begin{array}{l}\text { It was interesting to go through each } \\
\text { module }\end{array}$ & $4(4-5)$ \\
\hline 5. & Videos were of very good quality & $4(4-5)$ \\
\hline 6. & Videos explained the practical aspects well & $4(4-5)$ \\
\hline 7. & Entire content was well organized & $4(4-5)$ \\
\hline \multicolumn{3}{|c|}{$\begin{array}{l}\text { Teaching methodology used for updating CPR } \\
\text { guideline }\end{array}$} \\
\hline 8. & $\begin{array}{l}\text { It was convenient for me to go through the } \\
\text { entire training module in the stipulated } \\
\text { time }\end{array}$ & $4(2-5)$ \\
\hline 9. & $\begin{array}{l}\text { Online chat helped me to clear my doubts } \\
\text { about the changes introduced in CPR due } \\
\text { to COVID-19 }\end{array}$ & $4(4-5)$ \\
\hline
\end{tabular}

enrollment were provided with a unique login ID and password (PW) to access the training modules (Supplementary Figs 1 and 2).

There was a significant improvement in the mean knowledge scores of nurses following the online intervention (13.65 \pm 3.01 vs $19.92 \pm 1.94, p=0.001)$. Most nurses had high satisfaction (67.12\%) with the content and the methodology used for imparting the training with the mean satisfaction scores of $37.23 \pm 4.70$ (Table 2).

Nurses found the modules well-organized, comprehensive, well-explained, easy to understand, interesting as evident by the median (IQR) scores of 4 (4-5) (Table 3); however, for a few nurses (5), it was relatively less convenient to complete the modules in the stipulated time.

\section{Discussion}

Our study revealed that the online CPR training program was effective in updating the knowledge of the nurses related to new CPR guidelines. Nurses were satisfied with the training methodology as well as the content of the training program.

Due to highly infectious disease, special precautions are to be observed while resuscitating a COVID-19 patient. $^{5-7}$ During the
COVID-19 pandemic, updating healthcare professionals using an online platform has become a globally tested method of teaching and learning. ${ }^{8-12}$ Electronic learning through an interactive medium like mobile phones or laptops is becoming popular among on-job learners. ${ }^{13,14}$ All HCP including nurses are expected to upgrade their knowledge, skills, and attitude for providing patients care during the pandemic. Keeping in view the COVID-19 situation, where there was no possibility of having close contact among the instructors and the participants in a real classroom like situation, we decided to use a self-paced online method for updating the CCLS-trained nurses with new CPR guidelines. In the present study, relatively low median scores of nurses on completion of the entire training module in the stipulated time shows that nurses need more time to get tuned to online learning. In the present study, researchers could not correlate the knowledge scores with the variants in demographic data such as age, gender, qualification, computer literacy, etc. Prior to this study, all the nurses had classroom experience of attending the CPR training, which is a psychomotor skill learning program. It is a universal fact that psychomotor skills can be best learned by doing. For attaining these skills, the learner has to go through different stages of psychomotor learning in a step-wise manner. ${ }^{14-16}$ In the present study, we could help the nurses attain the second or third stage of psychomotor learning, in which the nurses commented while seeing the teacher demonstrating. The e-learning can act as a foundation stone and prime the learners in new CPR guidelines before actual hands-on sessions. However, this e-learning program is not appropriate for the training of HCW who have never been exposed to the concept of CPR. ${ }^{16}$ At the end of the training program, all nurses were asked to revise their skills using simulated scenarios in their respective clinical areas by organizing periodic drills. The use of multimedia and interactive sessions in this training helped nurses in assimilating the knowledge and promote critical thinking. In the present study, the majority of nurses had expressed a high level of satisfaction with the training program including the methodology used. The course content was meticulously prepared using short webinars with embedded videos, keeping in mind the busy schedule of the nurses and the principles of adult learning, who could watch the webinars at their convenience. The training content was broken into mini capsules for a better understanding of the concepts and skills. They were given enough time to churn the gained knowledge before its assimilation. The entire training was made interactive by allowing nurses to post their queries in a WhatsApp group and arranging two online interactive virtual tutorials. The PDF reading material acted as a foundation on which all the videos were based to enrich the experience of the learners. Some of the nurses (25\%) have recommended incorporating a small self-marking quiz before and after the training the completion of each webinar to consolidate their learning on the topic. All nurses 
found the training program useful, just on-time learning during the COVID-19 pandemic. There was no specified time zone to access the wealthy resource material on CCLS. The present study has some limitations too. A single center study, self-report satisfaction, and the absence of a control group limits the generalizability of the study. There was no long-term follow-up done to determine the retention and continuum of use of newly acquired knowledge. We could not assess the effectiveness of the training program in terms of new skills acquisition and positive impact on COVID-19 patients' well-being. There is a need to replicate the study in a larger population and assess the impact of training on clinical outcome of the patients. The findings of the present study may be used an opportunity for conducting a future training program for those could not attend the first round of the training. This online method of dissemination of CPR guidelines is restricted to the settings having resources for online training. This is a timely piece of research during COVID pandemic used for training the nurses in treating COVID-19 patients is an investment in the cause of medicine at large.

\section{CONCLUSION}

Well-structured, online study material was effective in imparting new knowledge and understanding the basic and essential skills required for giving CPR to COVID-19 patients.

\section{ORCID}

Poonam Joshi @ https://orcid.org/0000-0002-7016-8437

Smita Das $\odot$ https://orcid.org/0000-0002-4157-601X

Merin Thomas ㄴ https://orcid.org/0000-0003-3482-7199

Shashi Mawar @ https://orcid.org/0000-0002-4784-2980

Rakesh Garg 1 https://orcid.org/0000-0001-5842-8024

Ahamadulla Shariff $\odot$ https://orcid.org/0000-0002-9201-8544

Lakshmanan Gopichandran @ https://orcid.org/0000-0003-2738-6030

\section{Supplementary Material}

All the supplementary Figures 1 and 2 are available online on the website of www.IJCCM.org

\section{References}

1. Singh B, Garg R, Rao SSCC, Ahmed SM, Divatia JV, Ramakrishnan TV, et al. Indian resuscitation council (IRC) suggested guidelines for comprehensive cardiopulmonary life support (CCLS) for suspected or confirmed coronavirus disease (COVID-19) patient. Indian J Anaesth 2020;64(14):91. DOI: 10.4103/ija.IJA_481_20.

2. Wang J, Du G. COVID-19 may transmit through aerosol. Irish J Med Sci 2020;1-2. DOI: 10.1007/s11845-020-02218-2.

3. e-Learning as good as traditional training for health professionals: where is the evidence? WHO.

4. Vaona A, Banzi R, Kwag KH, Rigon G, Cereda D, Pecoraro V, et al. E-learning for health professionals. Cochrane Database Syst Rev 2018;1(1):CD011736. DOI: 10.1002/14651858.CD011736.pub2.

5. Judson SD, Munster VJ. Nosocomial transmission of emerging viruses via aerosol-generating medical procedures. Viruses 2019;11(10):940. DOI: $10.3390 / v 11100940$.

6. Centers for Disease Control and Prevention (CDC). Available from: https://www.cdc.gov/coronavirus/2019-ncov/hcp/infection-controlfaq.html [Accessed on May 9, 2020].

7. World Health Organization (WHO). Available from: https://apps. who.int/iris/bitstream/handle/10665/331695/WHO-2019-nCovIPC_PPE_use-2020.3-eng.pdf [Accessed on May 10, 2020].

8. McCutcheon K, Lohan M, Traynor M, Martin D. A systematic review evaluating the impact of online or blended learning vs face-to-face learning of clinical skills in undergraduate nurse education. J Adv Nurs 2015;71(2):255-270. DOI: 10.1111/jan.12509.

9. Jenkins JM, Cook J, Edwards J, et al. Medical education with the Internet: a pilot training programme in reproductive medicine. Br J Obstet Gynaecol 2001;108(1):114-116. DOI: 10.1111/j.14710528.2001.00010.x.

10. BMJ learning: online courses for healthcare professionals. Available from: https://new-learning.bmj.com/.

11. Ruiz JG, Mintzer MJ, Leipzig RM. The impact of E-learning in medical education. Acad Med 2006;81(3):207-212. DOI: 10.1097/00001888200603000-00002.

12. Sincliar P, Kable A, Levett-Jones T. The effectiveness of internet based e-learning on clinical behaviour and patient outcomes: a systematic review protocol. JBI Database System Rev Implement Rep 2015;13(1):52-64. DOI: 10.11124/jbisrir-2015-1919.

13. Thukral A, Sasi A, Chawla D, Datta P, Wahid S, Rao S, et al. Online Neonatal Training and Orientation Programme in India (ONTOP-IN) - the way forward for distance education in developing countries. J Trop Pediatr 2012;58(6):486-490. DOI: 10.1093/tropej/fms029.

14. Choules AP. The use of elearning in medical education: a review of the current situation. Postgrad Med J 2007;83(978):212-216. DOI: 10.1136/ pgmj.2006.054189.

15. Chan TW, Roschelle J, Hsi S, Kinshuk, Sharples M, Brown T, et al. One-to-one technology-enhanced learning: an opportunity for global research collaboration. Res Pract Technol Enhanc Learn 2006;1(1):3-29. DOI: 10.1142/S1793206806000032.

16. Osborne E. Teaching Strategies for Developing Psychomotor Skills. NACTA Journal 1986;30(1):54-57. Available from: http://www.jstor. org/stable/43755313, accessed on 10.05.21. 